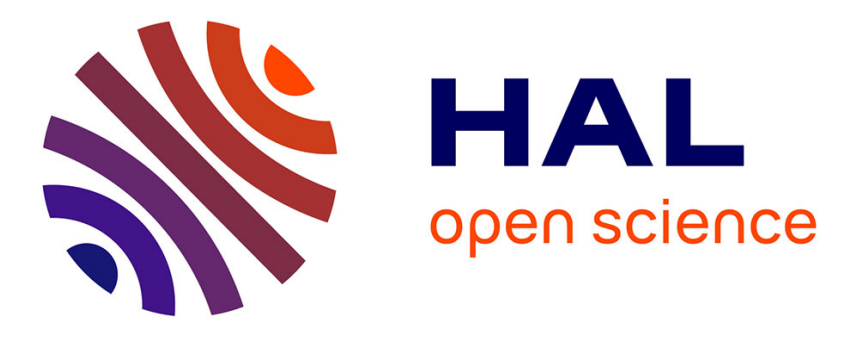

\title{
Outpatient vagus nerve stimulation surgery in drug-resistant epilepsy patients with severe intellectual disability
}

Insafe Mezjan, Isabelle Gourfinkel-An, Vincent Degos, Stéphane Clemenceau, Vincent Navarro, Véronique Masson, Alexandre Carpentier, Bertrand Mathon

\section{- To cite this version:}

Insafe Mezjan, Isabelle Gourfinkel-An, Vincent Degos, Stéphane Clemenceau, Vincent Navarro, et al.. Outpatient vagus nerve stimulation surgery in drug-resistant epilepsy patients with severe intellectual disability. Epilepsy \& Behavior, 2021, 118, pp.1-4. 10.1016/j.yebeh.2021.107931 . hal-03263469

\section{HAL Id: hal-03263469 \\ https://hal.sorbonne-universite.fr/hal-03263469}

Submitted on 17 Jun 2021

HAL is a multi-disciplinary open access archive for the deposit and dissemination of scientific research documents, whether they are published or not. The documents may come from teaching and research institutions in France or abroad, or from public or private research centers.
L'archive ouverte pluridisciplinaire HAL, est destinée au dépôt et à la diffusion de documents scientifiques de niveau recherche, publiés ou non, émanant des établissements d'enseignement et de recherche français ou étrangers, des laboratoires publics ou privés. 
Outpatient vagus nerve stimulation surgery

in drug-resistant epilepsy patients with severe intellectual disability

Insafe MEZJAN, MD ${ }^{1}$; Isabelle GOURFINKEL-AN, MD, PhD ${ }^{2,3}$; Vincent DEGOS, MD, PhD $^{4,5,6}$; Stéphane CLEMENCEAU, MD'; Vincent NAVARRO, MD, PhD ${ }^{2,3,7}$; Véronique MASSON ${ }^{2}$; Alexandre CARPENTIER, MD, PhD ${ }^{1,7}$; Bertrand MATHON, MD ${ }^{1,7}$.

1. APHP, Department of Neurosurgery, Sorbonne University, La Pitié-Salpêtrière Hospital, Paris, France.

2. APHP, Department of Neurology, Epileptology Unit, Sorbonne University, La PitiéSalpêtrière Hospital, Paris, France

3. APHP, Reference Center for Rare Epilepsies, Sorbonne University, La PitiéSalpêtrière Hospital, Paris, France

4. APHP, Department of Anesthesia, Critical Care and Peri-Operative Medicine, PitiéSalpêtrière Hospital, Sorbonne University, Paris, France.

5. Clinical Research Group ARPE, Sorbonne University, Paris, France.

6. INSERM UMR 1141, PROTECT, Paris France.

7. Paris Brain Institute, INSERM, CNRS, Sorbonne University, UMR S 1127, Paris Brain Institute, ICM, F-75013, Paris, France.

Reprints and Correspondence: Bertrand Mathon, MD, MSc, Department of Neurosurgery, La Pitié-Salpêtrière University Hospital, 47-83, boulevard de l'Hôpital, 75651 Paris Cedex 13, France. Tel: +33 (0)1 848273 63; e-mail: bertrand.mathon@aphp.fr 


\section{ABSTRACT}

Purpose: Vagus nerve stimulation (VNS) implantation is increasingly proposed in outpatient procedure. Some epilepsy syndromes are associated with severe neurodevelopmental disabilities (intellectual disability, autism) and often motor or sensory handicaps, making ambulatory surgery more complex.

Methods: We prospectively assessed the feasibility and safety of outpatient VNS implantation in 26 adult drug-resistant epilepsy patients with severe intellectual disability between December 2017 and October 2020.

Results: The male-to-female ratio was 0.9 and the mean age on surgery day was 23.1 years. Seventeen patients (65.4\%) suffered from epileptic encephalopathy, 7 (26.9\%) from cryptogenic or genetic generalized epilepsy and 2 (7.7\%) from severe multifocal epilepsy. Postoperatively, all patients discharged the day of surgery. No patient was admitted to a hospital or have consulted within one month due to postoperative complications. No surgeryrelated complication underwent during the patients' follow-up.

Conclusion: Our study highlights the safety and feasibility of VNS surgery in an outpatient setting for patients with severe intellectual disability. We report detailed protocol and preoperative checklist to optimize outpatient VNS surgery in these not able-bodied patients. Severe disabilities or epilepsy-associated handicaps should not be an exclusion criterion when considering ambulatory VNS implantation.

\section{KEYWORDS}

Ambulatory; epilepsy surgery; epileptic encephalopathy; postoperative management; drugresistant epilepsy; multiple disabilities. 


\section{INTRODUCTION}

Epilepsy affects $1 \%$ of the population and one-third of patients have seizures refractory to pharmacotherapy.[1] Vagus nerve stimulation (VNS) has been part of the surgical management for drug-resistant epilepsy for over 20 years. Following VNS, about $50 \%$ to $60 \%$ of patients achieve $50 \%$ reduction in seizure frequency and $8 \%$ show complete seizure freedom.[1,2]

VNS implantation is increasingly proposed in outpatient procedure.[3] In our institution, VNS implantation was performed on outpatient procedure as the standard of care for ablebodied patients since March 2017.

Some epilepsy syndromes are associated with intellectual disabilities and multiple handicaps, making ambulatory surgery more complex. However, the families and guardians of these patients usually request outpatient care to avoid disturbance of their emotional and behavioral balance.

We aimed to assess the feasibility and safety of outpatient VNS implantation in multiply handicapped patients.

\section{METHODS}

\subsection{Patient selection and study design}

We enrolled all severely handicapped patients over the age of 18 years with drugresistant epilepsy who were referred for VNS surgery at the Pitié-Salpêtrière University Hospital (Paris, France) between December 2017 and October 2020. The exclusion criteria for ambulatory VNS implantation were i) whether the parent or legal guardian has refused an outpatient management, ii) whether the patient had medical comorbidity necessitating more than 6 hours of postoperative observation (e.g. hemostasis disorders) and iii) whether the patient was not living within a radius of less than one hour of transport of the hospital. 
All patients underwent epileptological evaluations in our Reference Center for Rare Epilepsies. These data were analyzed by a multidisciplinary team to determine whether VNS was indicated. The following variables were registered: age, sex, American Society of Anesthesiologists class (ASA 1-5), medication(s), time from initiation to completion of surgery, time from initiation to completion of anesthesia, time from completion of anesthesia to discharge, modified post anesthetic discharge scoring system (mPADS, Fig. 1), discharge to home, institution, or hospital, hospital admission within one month after surgery, unplanned consultation within one month after surgery, surgery-related complication and thromboembolism presenting within one month after surgery, persistent dysphagia and hoarseness at six weeks.

\subsection{Preoperative period, day surgery and postoperative management (Fig.1)}

All instructions were prior given during a preoperative therapeutic education consultation conducted by a specialty nurse in the presence of the patient, his legal representatives and often member of his institution (Table 1). All VNS surgeries were scheduled for the morning in order to allow for sufficient postoperative observation and discharge before $7 \mathrm{pm}$ on the day of surgery. A povidone-iodine shower was performed in the morning of the intervention at home and patient was admitted in the morning in the outpatient surgery unit. To decrease surgery induced patient's stress, the guardian was present in the operating room until the anesthesia induction was complete, as well as in the recovery unit after the surgery. The procedure was performed under general anesthesia as previously described.[4] The patient was then observed for at least 2 hours in a recovery unit.

A snack was provided to the patient upon return to the outpatient surgery unit. Patient was discharged if the following modified clinical discharge criteria (mCDC, Fig.1)[5] were satisfactory. Precautionary instructions and warning signs as well as emergency call numbers were given to the guardian on discharge (Table 1). The guardian was strongly advised to closely monitor the patient the first night after surgery. One of the board-certified neurosurgeons can be contacted by mobile phone 24 hours a day, 7 days a week and guardian 
was requested to call the hospital with any question or concerns. Neurosurgeon's email address was also provided to the guardian in case of doubt on wound healing.

The day after surgery the guardian was contacted by a specialty trained nurse. If the patient had problems or complaints, he received further supervision. Three weeks after surgery, the patient consulted the neurologist to turn the stimulator on and adjust stimulation parameters. A follow-up neurosurgical consultation was routinely done 6 weeks after surgery during which guardian's satisfaction about outpatient management was assessed.

\subsection{Judgement criteria}

The primary endpoints were i) an unplanned hospitalization following VNS surgery, ii) an unplanned hospitalization or consultation related to VNS surgery within one month of surgery and iii) surgery-related complications. The secondary endpoint was the guardian's satisfaction scale (from very dissatisfied to very satisfied).

\subsection{Standard protocol approvals}

The database is registered with the Commission Nationale de I'Informatique et des Libertés ( $\left.\mathrm{n}^{\circ} .2214386\right)$. In accordance with the ethical standards of our hospital's institutional review board, written informed consent was obtained from all patients' guardians. The manuscript was prepared in accordance with the STrengthening the Reporting of OBservational studies in Epidemiology (STROBE) statement. 


\section{RESULTS}

\subsection{Patients' characteristics}

During the study period, 74 patients underwent VNS surgery, out of which 34 had severe intellectual disability. Eight did not meet the above-mentioned prespecified criteria: four patients were living too far from hospital, 3 families refused the protocol and one had hemostasis disorders. Twenty-six patients $(76.5 \%)$ were eligible for outpatient management and were included in the study.

The male-to-female ratio was 0.9 and the mean age on surgery day was $23.1+/-6.7$ years. Seventeen patients (65.4\%) suffered from epileptic encephalopathy, 7 (26.9\%) from drug-resistant cryptogenic or genetic generalized epilepsy and $2(7.7 \%)$ from severe multifocal epilepsy.[6] Twenty-two patients (84.6\%) were treated with three or more antiepileptic drugs at the time of surgery. All patients had severe cognitive and behavioral disabilities, have been placed under extended guardianship and were living in institutions. All patients except one were classified as ASA 3 during the pre-anesthesia consultation.

\subsection{Patients' outcomes}

Operative and peri-operative durations are summarized in Fig.1. During the postoperative observation, the $\mathrm{mCDC}$ have been reached for all patients enabling discharge to home $(71.4 \%)$ or to institution $(28.6 \%)$ the day of surgery. Patients who were discharged to home came back to their institution within days (range 1 - 7 days) according to the patient's family wish. The mPADS score for assessing patient discharge ability was $8 / 8$ in 25 patients $(89.3 \%)$ and $7 / 8$ in 3 patients $(10.7 \%)$. No patient was admitted to a hospital or consulted within one month due to postoperative complications. In addition, no surgery-related complication underwent during the patients' follow-up.

Out of 22 guardians, $77.3 \%$ were very satisfied and $22.7 \%$ were satisfied with the outpatient management. 


\section{DISCUSSION}

In our series, 26 drug-resistant epilepsy patients with severe intellectual disability underwent a VNS implantation in an outpatient setting and all were successfully discharged 4 hours after the procedure. No patient required an unplanned consultation or rehospitalization. There were no complications or negative outcomes as a result of early discharge. The satisfaction rate from the guardians as regards to the same-day discharge was excellent. Thus, our study confirms the safety and feasibility of VNS implantation in patients with intellectual disability in an outpatient setting.

Improvement in perioperative care has led to earlier discharge and to the development of outpatient procedures in neurosurgery. In addition to its medical and psychological advantages for the patient, outpatient neurosurgery optimizes health care costs and hospital bed flow.[7] VNS implantation is performed as a day-surgery in $79.5 \%$ of cases in the USA [8] but less performed procedure in patients with disabilities.

We strongly believe that ambulatory surgery is a valuable strategy for patients with intellectual disability. An overnight stay in the hospital can easily disturb their emotional and behavioral balance.[9] Furthermore, hospital-based complications such as nosocomial infection and medical error are avoided, which is crucial for these vulnerable patients with complex condition and treatments. We experienced no surgery-related complication in our series and no patient required an unplanned consultation or rehospitalization. Our results confirm the low rate of complication previously mentioned in the literature. Indeed, Kalanithi et al. reported a one-year reoperation rate of $3.9 \%$ and a complication rate within the first 30 days $<2 \%$.[8] The most common surgical complications being hoarseness, voice alteration, dyspnea and postoperative pain.[10] Because infirmities and intellectual disability are usually exclusion criteria for outpatient surgery, we found no data in the literature concerning VNS implantation as day-case surgery in severely handicapped patients. To our knowledge, our study is the first one focusing on VNS implantation as an outpatient surgery in this particular population.

Multiple factors may explain our high discharge rate and lack of complication: 
-The existence of an outpatient surgery unit in our institution with a dedicated and experienced team[11] and clearly established protocols (Table 1).

-The close cooperation between neurosurgeons, anesthesiologists and the ambulatory surgery unit nurses was established prior to the study. Indeed, ambulatory VNS implantation became the standard of care for able bodied patients nine months prior to the inclusion of severely handicapped patients.

-The need for an appropriate discharge environment: two of our inclusion criteria being living within a radius of less than one hour of transport of the hospital and the need for the patient's guardian to be present and available to care for the patient after the procedure. The caregivers are appropriately educated on the possible complications and their early signs. Because the primary family caregivers are critical partners in the plan of care for severely handicapped patients, they have often acquired the skills and knowledge necessary to appropriately care for the patient. They are usually in tune with the patient's needs and comfort making them the best caregiver possible for the patient. After VNS surgery, patients can thereby discharge from hospital to home or to their institution depending on both the family's preference and the home environment.

-To minimize the infection risk, we make a reinforced initial wound bandage (Fig.1) and prescribe daily wound care by a nurse at home until complete healing. For the patient's comfort, the closure of the skin wound is done using absorbable suture thread.

Our outpatient protocol is met with a high rate of satisfaction from the patients' guardians. This is consistent with reports of high patient satisfaction following various brain and spine surgery in an outpatient setting.[12] Guardians' satisfaction in our study is in part due to the guardian-centered strategy we adopted to minimize the patient's anxiety. To help the patient cope with the stressful environment, the guardian is present in the operating theater until the anesthesia induction is complete, as well as in the recovery unit after the procedure. This is in line with the benefits of a discharge on surgery-day as it allows the patients to recover in a familiar environment with assistance and observation provided by their usual caregivers. This effectively reduces the psychological impact of a hospitalization in this fragile population. 
Our study has some limitations. First, it is monocentric, observational design, including a small number of patients. Second, our institution being a reference center for rare epilepsies induces a selection bias, but also reflects the real-world picture of drug-resistant epilepsy patients with severe intellectual disability. Third, the guardian's satisfaction evaluation is based on a subjective and qualitative questionnaire. Further studies should assess guardian's satisfaction using objective measurements and/or validated form. Last, outpatient management in our center relies on the extensive experience of our team, and those results may not be immediately reproducible in every center that are not already familiar with the outpatient management of able-bodied patients.

\section{CONCLUSION}

Our study highlights safety and feasibility of VNS surgery in an outpatient setting among 26 patients with severe intellectual disability. Developmental disability or associated multiple handicaps should not be an exclusion criterion when considering ambulatory VNS implantation. Development of this outpatient procedure makes it possible to offer VNS surgery to more patients in this particular group whose families may be reluctant to traditional hospitalization in the neurosurgery department.

\section{ACKNOWLEDGMENTS}

The authors thank Dr. Steffen Fetzer for advice and discussion concerning the manuscript.

\section{ETHICAL PUBLICATION STATEMENT}


We confirm that we have read the Journal's position on issues involved in ethical publication and affirm that this report is consistent with those guidelines. 


\section{LEGENDS}

Fig. 1. Timeline of outpatient vagus nerve stimulation surgery in drug-resistant epilepsy patients with severe intellectual disability.

Surgery hours are provided for guidance purposes only for a patient operated on the first position. The occlusive bandage is designed in such a way that the patient cannot access to his wound. This reinforced bandage can be lightened the day after surgery. Nursing care: A nurse makes daily wound care at patient's home until complete healing. In case of abnormality or doubt on wound healing, he/she would be asked to send a picture of the wound by email directly to the neurosurgeon.

D, day; OR, operating room; VNS, vagus nerve stimulation. Durations are presented as mean $(\min -\max )$.

Table 1. Preoperative checklist for outpatient vagus nerve stimulation surgery in drugresistant epilepsy patients with severe intellectual disability. 


\section{REFERENCES}

[1] Englot DJ, Rolston JD, Wright CW, Hassnain KH, Chang EF. Rates and Predictors of Seizure Freedom With Vagus Nerve Stimulation for Intractable Epilepsy. Neurosurgery 2016;79:345-53. https://doi.org/10.1227/NEU.0000000000001165.

[2] González HFJ, Yengo-Kahn A, Englot DJ. Vagus Nerve Stimulation for the Treatment of Epilepsy. Neurosurg Clin N Am 2019;30:219-30. https://doi.org/10.1016/j.nec.2018.12.005.

[3] Mallereau C-H, Ollivier I, Valenti-Hirsch M-P, Hirsch E, Proust F, Chaussemy D. Vagus nerve stimulation in epilepsy: Efficiency and safety of outpatient practice. Neurochirurgie 2020;66:270-4. https://doi.org/10.1016/j.neuchi.2020.04.134.

[4] Giordano F, Zicca A, Barba C, Guerrini R, Genitori L. Vagus nerve stimulation: Surgical technique of implantation and revision and related morbidity. Epilepsia 2017;58 Suppl 1:85-90. https://doi.org/10.1111/epi.13678.

[5] Chung F, Chan VW, Ong D. A post-anesthetic discharge scoring system for home readiness after ambulatory surgery. J Clin Anesth 1995;7:500-6. https://doi.org/10.1016/0952-8180(95)00130-a.

[6] Scheffer IE, Berkovic S, Capovilla G, Connolly MB, French J, Guilhoto L, et al. ILAE classification of the epilepsies: Position paper of the ILAE Commission for Classification and Terminology. Epilepsia 2017;58:512-21. https://doi.org/10.1111/epi.13709.

[7] Marigil M, Bernstein M. Outpatient neurosurgery in neuro-oncology. Neurosurg Focus 2018;44:E19. https://doi.org/10.3171/2018.3.FOCUS1831.

[8] Kalanithi PSA, Arrigo RT, Tran P, Gephart MH, Shuer L, Fisher R, et al. Rehospitalization and emergency department use rates before and after vagus nerve stimulation for epilepsy: use of state databases to provide longitudinal data across multiple clinical settings. Neuromodulation J Int Neuromodulation Soc 2014;17:60-4; discussion 64-65. https://doi.org/10.1111/ner.12051.

[9] Pellock JM, Morton LD. Treatment of epilepsy in the multiply handicapped. Ment Retard Dev Disabil Res Rev 2000;6:309-23. https://doi.org/10.1002/10982779(2000)6:4<309::AID-MRDD10>3.0.CO;2-I.

[10] Pérez-Carbonell L, Faulkner H, Higgins S, Koutroumanidis M, Leschziner G. Vagus nerve stimulation for drug-resistant epilepsy. Pract Neurol 2020;20:189-98. https://doi.org/10.1136/practneurol-2019-002210.

[11] Gilmartin J, Wright $K$. The nurse's role in day surgery: a literature review. Int Nurs Rev 2007;54:183-90. https://doi.org/10.1111/j.1466-7657.2007.00528.x. 
[12] Outpatient neurosurgery: Expert Review of Neurotherapeutics: Vol 16, No 4 n.d. https://www.tandfonline.com/doi/full/10.1586/14737175.2016.1158104 (accessed January 3, 2021). 